\title{
Neurokognitive und psychiatrische Störungen
}

\section{Liebe Leserinnen, liebe Leser,}

in unserer modernen Welt, in der der Anteil alter und multimorbider Menschen ständig zunimmt und die soziale und medizinische Versorgung dieser Menschen eher schlechter wird, muss das Hauptaugenmerk auf die Prävention gelegt werden. Dies gilt auch oder gerade für neurokognitive und neurodegenerative Störungen und Erkrankungen.

Unsere Kenntnis über die Ursachen und auch über verschiedene Interventionsmöglichkeiten dieser Erkrankungen hat in den letzten Jahren stark zugenommen. Ursächliche Zusammenhänge mit Ernährungsfaktoren bzw. Nährstoffen, aber auch mit dem Ausmaß der (geistigen/koordinativen) Aktivität sind unübersehbar. In diesen Bereichen sollten präventive Maßnahmen ansetzen.

Vor dem Hintergrund unserer „Mangelernährung im Überfluss“ (Mangel an wichtigen Nährstoffen, Überfluss an Energieträgern und schädlichen Substanzen) muss die präventive Intervention schon vor der Geburt ansetzen. So ist z.B. eine ausreichende Versorgung mit DHA (Docosahexaensäure) eine
Grundvoraussetzung für eine ungestörte Entwicklung und Reifung des Gehirns. Allerdings ist die Versorgung der Mütter mit dieser wichtigen Fettsäure eher schlecht, sodass der Mangel und damit das Risiko einer suboptimalen Hirnentwicklung des Kindes bereits programmiert ist. Ein optimal entwickeltes und ohne jede Einschränkung (d.h. maximale Plastizität und Verschaltung) funktionierendes und ständig aktiv gefordertes Gehirn kann eventuelle Störungen im Alter ganz anders kompensieren als ein Gehirn mit bereits seit der Entwicklungsphase bestehenden Einschränkungen (ohne dass diese klinisch sichtbar sind!), das im Laufe des Lebens weniger durch Aktivität gefordert wird.

Die Bedeutung von oxidativem Stress und chronischer Entzündung (chronic low level inflammation) als Ursache degenerativer Prozesse ist ausreichend bekannt und gilt auch für den Bereich neurokognitiver und neurodegenerativer Störungen und Erkrankungen. Dies sollte im Rahmen der Prävention besonders berücksichtigt werden und seinen Nie- derschlag in einer entsprechenden Ernährung und wo sinnvoll auch in einer Substitution mit Mikronährstoffen und pflanzlichen Substanzen finden.

Prävention ist umso wirksamer, je früher sie einsetzt. Zu warten, bis immer mehr Menschen aufgrund ihrer Hirnleistungsstörungen $\mathrm{zu}$ einem sozialen und medizinischen Problem geworden sind, das dann mit pharmazeutischen Präparaten und/oder Verwahrung in „Pflegeheimen“ gelöst werden soll, kann nicht der richtige Weg sein - auch wenn dieser Weg inzwischen einen nicht unbedeutenden Wirtschaftsfaktor darstellt.

Ihre Herausgeber

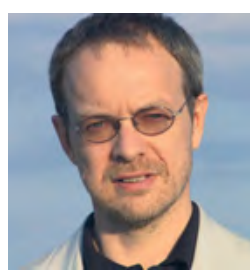

Dr. med. Hans-Peter Friedrichsen

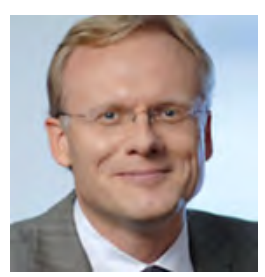

Apotheker Uwe Gröber 